\title{
Absolute Fluorescence Calibration
}

\author{
I.T. Young ${ }^{*}$, Y. Garini, B. Vermolen, G. Liqui Lung, G. Brouwer, S. Hendrichs, M. el Morabit, J. \\ Spoelstra, E. Wilhelm, M. Zaal
}

\section{Quantitative Imaging Group, Department of Imaging Science \& Technology, Faculty of Applied Sciences, Delft University of Technology, Lorentzweg 1, NL-2628 CJ Delft, The Netherlands}

\begin{abstract}
While fluorescence microscope systems remains an essential tool in modern biology and medical work, no compact instrumentation has been developed for the rapid calibration of such systems. Almost invariably results are presented in terms of the [AU], "arbitrary units". To remedy this situation we have developed a small, portable instrument - the size of a microscope slide - that uses low-power LEDs at different wavelengths to produce calibrated amounts of light. A computer controls the instrument-through a USB connector-so that the current to the selected LED can be swept through an increasing range of values. The amount of light measured by the microscope's total imaging system (lenses, filters, EO sensor, and digitizer) is then recorded to provide a "current in, digital value out" calibration. Further, the current can be translated easily to optical power and thus photons per second at the chosen LED wavelength. We have built and programmed such a system, tested it for accuracy and precision, and used it to calibrate several microscopes and microscope/lens combinations. The results will be presented.
\end{abstract}

Keywords: fluorescence calibration, fluorescence microscopy, LED sources, quantitative microscopy

\section{INTRODUCTION}

Modern cell research is dependent upon the use of fluorescence techniques to make cells and cellular components visible. Gene probes, immunofluorescent labels, and fluorescent proteins are intimately associated with the study of cell structure, function, and evolution. Yet virtually all results that are reported in the literature are presented as comparative qualitative data. As illustrated in Figure 1, the units that are almost invariably used to describe fluorescent measurements are $[\mathrm{AU}]$, arbitrary units.

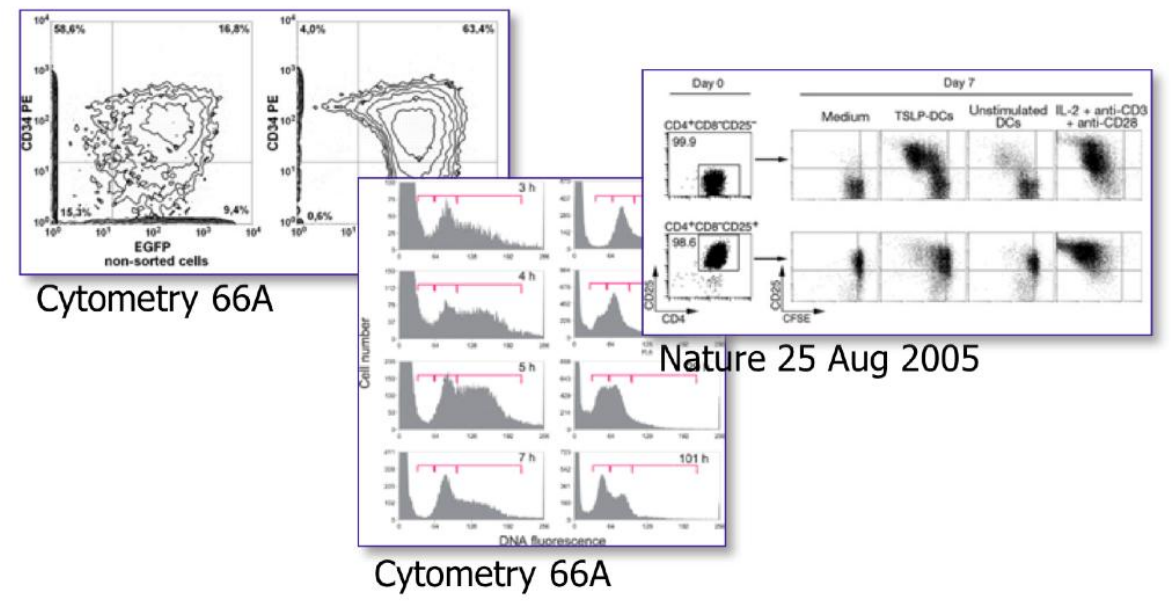

Figure 1: Examples of recent publications presenting fluorescence measurements as "arbitrary units". Left -Lennartz, et al. ${ }^{1}$, Center - Vogt, et al. ${ }^{2}$, Right - Watanabe, et al. ${ }^{3}$

\footnotetext{
* i.t.young <at> tudelft < dot> nl; phone +31-15-278-1416; fax +31-15-278-6740; http://www.qi.tnw.tudelft.nl/
} Robert C. Leif, Proc. of SPIE Vol. 6088, 60880U, (2006) · 1605-7422/06/\$15 · doi: 10.1117/12.638559 
It is clear that a simple, reliable, inexpensive procedure to provide absolute calibration of fluorescence is desirable. This would make it possible to compare easily measurements that were made on two different systems where "different" should be interpreted in the broadest possible sense. Measurements made in two different laboratories fall into this category as well as measurements made at two different times on the same system as well as measurements made with varying configurations on the same system. Three possible ways to achieve this are:

1) Number of fluorescing molecules

- This is a seemingly ideal goal and, indeed, polystyrene spheres coated with specific numbers of fluorescing molecules can be purchased from companies such as Becton Dickinson Biosciences. (See QuantiBRITE PE beads at http://www.bdbiosciences.com/).

2) Number of electrons involved in a standard light source - Light sources are powered by electricity and the technology for generating known, repeatable amounts of current has been worked out in detail. It is therefore possible to produce a light source that mimics a fluorescent cell (or cellular component) where the amount of light is known in absolute terms through the current driving the light source.

3) Number of photons produced in a standard light source - It is possible to determine the number of photons that are being emitted by a light source through straightforward use of basic principles. The basis for this will be developed later in this manuscript.

Of these three alternatives, we choose for the last two as they offer a portable, inexpensive, simple, and automated way to calibrate fluorescent microscopes.

\section{MATERIALS}

The light sources that we use to mimic fluorescing cells are low-power, light-emitting diodes (LEDs). The light source is constructed on a printed-circuit board whose dimensions are comparable to a microscope slide, $25 \mathrm{~mm} \mathrm{x} 75 \mathrm{~mm}$. In fact, eight LEDs can be mounted on this board as illustrated in Figure 2. All of the electronics are low power and low voltage. The connection to the computer is through a standard USB interface.
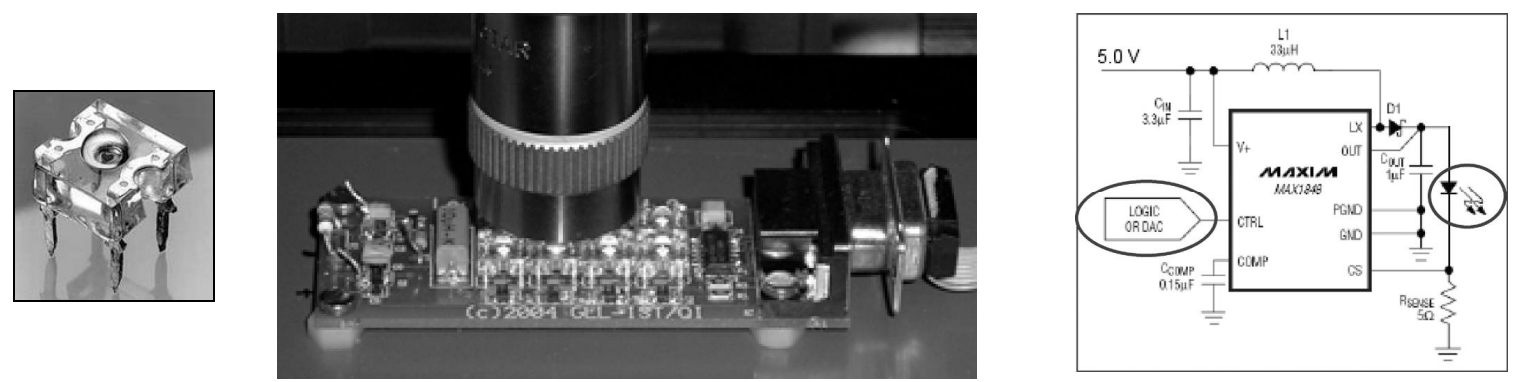

Figure 2: Left - Single Lumiled Superflux LED; Center - LED slide under microscope objective; Right - Digital controller that provides current LED which is regulated to $0.05 \%$.

The LEDs are all Lumiled Superflux light-emitting diodes with a maximum input power per LED of approximately 230 $\mathrm{mW}$, (Lumiled Lighting, http://www.lumileds.com/products/). Specifically, we have used the LEDS listed in Table 1.

Using an ASI spectral imager (Applied Spectral Imaging, <http://www.matimop.org.il/newrdinf/company/c2069.htm>, we measured the spectrum $E(\lambda)$ to determine the peak wavelength and the average wavelength for each LED type. A $500 \mathrm{~nm}$ narrow-band interference filter was used to check the spectral readout of the spectral imager. 
Table 1: LEDs used in the LED Microscope Slide

\begin{tabular}{|c|c|c|c|c|c|}
\hline $\begin{array}{c}\text { LED Light } \\
\text { Source }\end{array}$ & $\begin{array}{c}\text { Nominal } \\
\text { color }\end{array}$ & $\begin{array}{c}\text { Listed peak } \lambda \\
{[\mathrm{nm}]}\end{array}$ & $\begin{array}{c}\text { Measured peak } \\
\lambda[\mathrm{nm}]\end{array}$ & $\begin{array}{c}\text { Measured } \\
\text { average } \lambda[\mathrm{nm}]\end{array}$ & $\begin{array}{c}\text { Measured } \\
\text { FWHM [nm] }\end{array}$ \\
\hline HPWN MB00 & blue & 460 & 477 & 589 & 30 \\
\hline HPWN MC00 & cyan & 503 & 514 & 557 & 47 \\
\hline HPWN MG00 & green & 520 & 596 & 584 & 21 \\
\hline HPWT ML00 & amber & 596 & 619 & 613 & 24 \\
\hline HPWT MH00 & orange-red & 640 & 633 & 623 & 30 \\
\hline HPWT MD00 & red & & & & 4 \\
\hline
\end{tabular}

As is clear from Table 1, there can be a discrepancy between the "generic" peak wavelength listed by the manufacturer and the measured peak wavelength for a given LED. The normalized, measured spectra are shown in Figure 3.

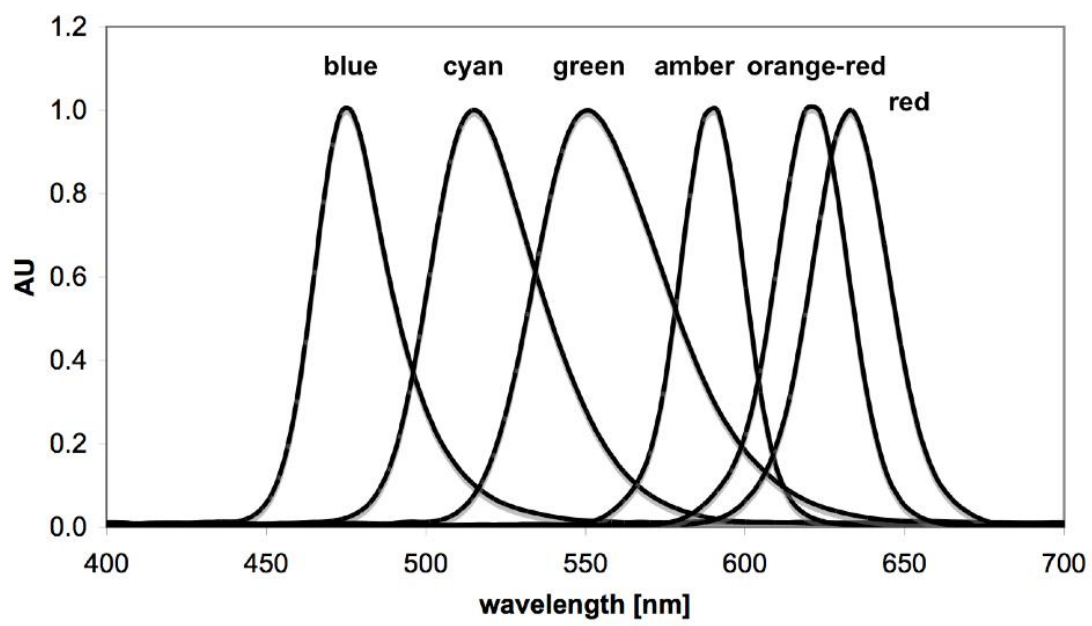

Figure 3: Normalized, measured $E(\lambda)$ for the six LEDs used in the calibration slide.

As these LEDs are mimicking fluorescent emission, it is useful to know which dyes can be calibrated according to these data. Table 2 presents a collection of fluorescent dyes whose emission wavelengths are within the FWHM of the peak wavelengths as measured in Figure 3 and presented in Table 1.

The extensive list of dyes makes it clear that there is a broad range of applicability of these six LEDs. Further, new LEDs with other peak wavelengths are becoming commercially available and it is to be expected that at some time in the near future virtually any wavelength in the range $450 \mathrm{~nm}$ to $700 \mathrm{~nm}$ will be available. See, for example, Farnell Electronics at $<\mathrm{http}: / / \mathrm{www}$.farnell.com/>.

The remainder of the measurement/calibration system requires a stabilized power supply to the LED Microscope Slide, an ordinary personal computer with a USB port, the microscope system including the microscope objectives, intermediary lenses, and the camera acquisition system that are to be calibrated. 
In the experiments described in this report, we have worked with a Leitz Aristoplan fluorescence microscope, an objective lens with $M=16 \times$ and $N A=0.45$, a Sony XC-77 video camera (768 x 576 pixels), and a Piccolo frame grabber. The software environment for the complete measurement/calibration procedure is MATLAB (MathWorks Inc., $<\mathrm{http}: / /$ www.mathworks.com/ $>$ ) with the addition of the DipImage image-processing suite as developed in our laboratories at the Delft University of Technology. (See <http://www.qi.tnw.tudelft.nl/DIPlib/>.)

Table 2: LED and fluorescent dye emission combinations suitable for the LED Microscope Slide. LED-Dye combinations are "suitable" when the peak, dye emission wavelength is within the FWHM of the measured peak LED wavelength (in parentheses).

\begin{tabular}{|c|c|}
\hline LED Light Source & Dye \\
\hline $\begin{array}{l}\text { HPWN MB00 blue } \\
\quad(477 \mathrm{~nm})\end{array}$ & $\begin{array}{l}\text { Marina Blue, DAPI, Hoechst 33258, SYTOX Blue, Indo-1, SYTO Blue BOBO-1, BO-PRO-1, } \\
\text { CFP }\end{array}$ \\
\hline $\begin{array}{l}\text { HPWN MC00 cyan } \\
\quad(514 \mathrm{~nm})\end{array}$ & $\begin{array}{l}\text { DIO, Fura-2, LysoSensor Green, YOYO-1, YO-PRO-1 } \beta \text {, LysoTracker Green, BODIPY FL, } \\
\text { SYTO Green, Fluo-4, MitoTracker Green, Calcein, FITC, Alexa 488, BCECF, ER Tracker Blue- } \\
\text { White DPX, Rhodamine } 110 \text {, SYTOX Green } \beta \text {, Oregon Green } 488 \text {, Alexa 500, JC-1, JC-9, } \\
\text { NeuroTrace 500/525, Acridine Orange, Fluo-3, Rhodamine Green, Rhodamine 123, ELF 97, } \\
\text { Oregon Green 514, Magnesium Green, Calcium Green, TOTO-1, TO-PRO-1 } \beta \text {, GFP, YFP }\end{array}$ \\
\hline $\begin{array}{l}\text { HPWN MG00 green } \\
\qquad(550 \mathrm{~nm})\end{array}$ & $\begin{array}{l}\text { Rhodamine Green, BCECF, Rhodamine } 123 \text {, ELF 97, Oregon Green 514, Magnesium Green, } \\
\text { Calcium Green, TOTO-1, TO-PRO-1 } \beta \text {, NBD amines, Sodium Green, Lucifer Yellow CH, Alexa } \\
\text { 430, Alexa 514, LysoSensor Yellow/Blue, SYTO Green, Eosin, Cascade Yellow, SYTO Orange, } \\
\text { Alexa 532, 6-Carboxyrhodamine 6G, JOE, Alexa 555, DiI, SYTOX Orange, Alexa 546, BODIPY } \\
\text { TMR-X, YFP }\end{array}$ \\
\hline $\begin{array}{l}\text { HPWT ML00 amber } \\
\qquad(591 \mathrm{~nm})\end{array}$ & $\begin{array}{l}\text { SYTO Orange, Resorufin, Carboxy SNARF, DiA, Lissamine rhodamine } \beta \text {, Rhodamine Red, } \\
\text { LysoTracker Red, JC-1, FM 1-43, MitoTracker Red CMXRos }\end{array}$ \\
\hline $\begin{array}{l}\text { HPWT MH00 } \\
\text { orange-red }(619 \mathrm{~nm})\end{array}$ & $\begin{array}{l}\text { Calcium Crimson, Texas Red, Alexa 594, BODIPY TR-X, EthD-1 } \beta \text {, Propidium iodide } \beta \text {, Nile } \\
\text { Red, YOYO-3, YO-PRO-3 } \beta\end{array}$ \\
\hline $\begin{array}{l}\text { HPWT MD00 red } \\
\quad(633 \mathrm{~nm})\end{array}$ & $\begin{array}{l}\text { Nile Red, YOYO-3, YO-PRO-3 } \beta \text {, SYTO Red, Carboxy SNARF, Di-8-ANEPPS, Di-4-ANEPPS, } \\
\text { JC-9, RH 414, Fura Red, 7-Aminoactinomycin D (7-AAD) } \beta \text {, Alexa 633, Acridine Orange }\end{array}$ \\
\hline
\end{tabular}

\section{METHODS}

The measurement procedure is as follows:

1) Choose / initialize the microscope, lens, camera, and computer system. This includes "parameters" such as filter choices, pixel binning, and integration time.

2) As the connection to the computer is via a USB port all programming can be accomplished with a simple set of ASCI strings. As mentioned above, we have used MATLAB as our software environment and programmed the ASCI interface accordingly.

3) Select the desired LED and set an intensity level that is $50 \%$ of the dynamic range. This corresponds to $300 \mu \mathrm{A}$ for the circuitry and the LEDs in our configuration.

4) Focus the image of the LED to establish a fixed axial height from the lens to the LED. A sample, focused image is shown in Figure 4.

5) Set the LED current to zero $(0 \mu \mathrm{A})$ and acquire a "black" image, $I_{\text {black }}(x, y)$,

6) Under computer control, step the LED current levels through $N$ different values and acquire an intensity image producing a set of images $\left\{I_{n}(x, y) \mid n=1,2,3, \ldots, N\right\}$. Typical values might be $N=30$ and a current increment of $20 \mu \mathrm{A}$ per step. In our facility this requires about $1 \mathrm{~s}$ per image for a total of about $30 \mathrm{~s}$. 
7) Computer the corrected, total brightness per image $B_{n}$ for each current level. This is defined as:

$$
\begin{aligned}
B_{n} & =\sum_{x} \sum_{y} I_{\text {corr }}(x, y)=\sum_{x} \sum_{y}\left\{I_{n}(x, y)-I_{\text {black }}(x, y)\right\} \\
& =\left(\sum_{x} \sum_{y} I_{n}(x, y)\right)-B_{0}
\end{aligned}
$$

where $B_{0}$ is the total intensity in the "black" image that needs to be computed only once.

8) Normalize $B_{n}$ to be the corrected intensity per pixel: $I_{n}=B_{n} /$ (pixels per image).

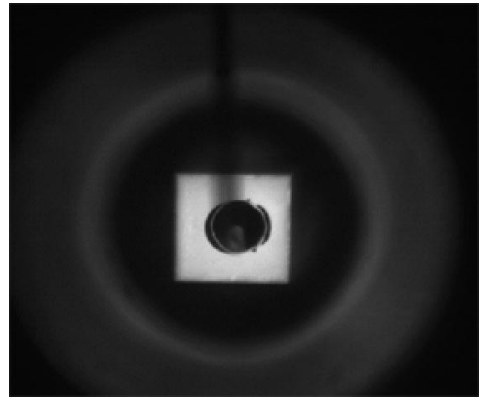

Figure 4: In-focus digital image of LED.

\section{EXPERIMENTAL RESULTS}

The results for three of the LEDs are shown in Figure 5. In the left and center graphs we see that the image intensity per pixel starts to saturate at the higher current levels. The rightmost graph is almost linear over the entire range of currents. Similar curves have been obtained for the three other LEDs.

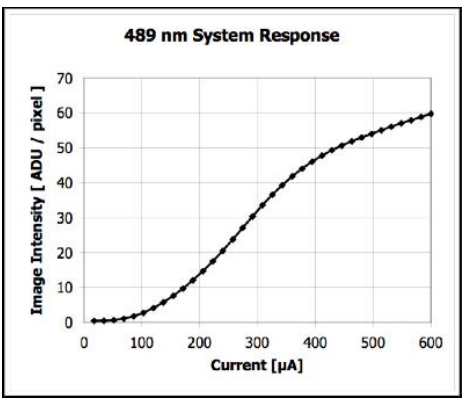

blue LED, $\lambda_{\text {peak }}=477 \mathrm{~nm}$

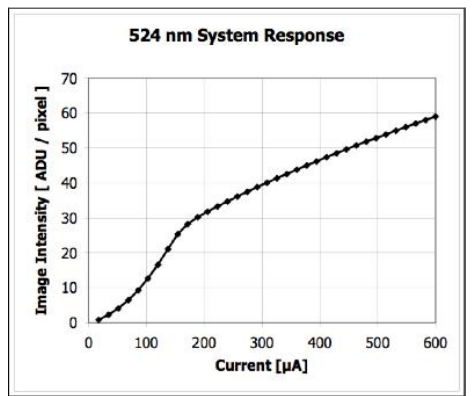

cyan LED, $\lambda_{\text {peak }}=514 \mathrm{~nm}$

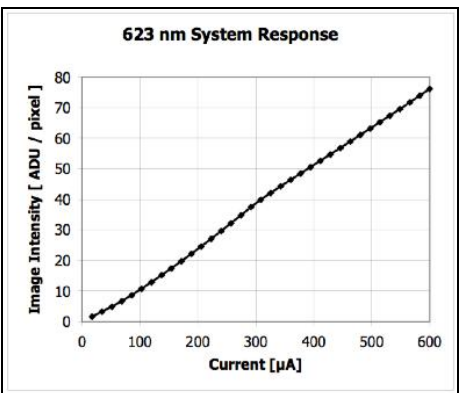

red $\mathrm{LED}, \lambda_{\text {peak }}=633 \mathrm{~nm}$

Figure 5: Measured intensity in image "grey values" versus current for three different LEDs. The measured average wavelength for each LED is indicated at the top of the graph.

As this equipment is to be used for absolute calibration, it is important to understand the stability of this procedure. To this end, we measured the calibration curve repeatedly over various intervals. Figure 6a shows the change in the "black" average image intensity per pixel over a time interval and Figure $6 \mathrm{~b}$ shows the variation in the calibration curve generated over the same interval. These data are generated from the cyan LED, $\lambda_{\text {peak }}=514 \mathrm{~nm}$ and $\lambda_{\text {avg }}=524 \mathrm{~nm}$ and the amber LED, $\lambda_{\text {peak }}=591 \mathrm{~nm}$ and $\lambda_{\text {avg }}=584 \mathrm{~nm}$. The cyan LED data were generated over a two-hour time interval and the amber LED data over a five-hour interval. 

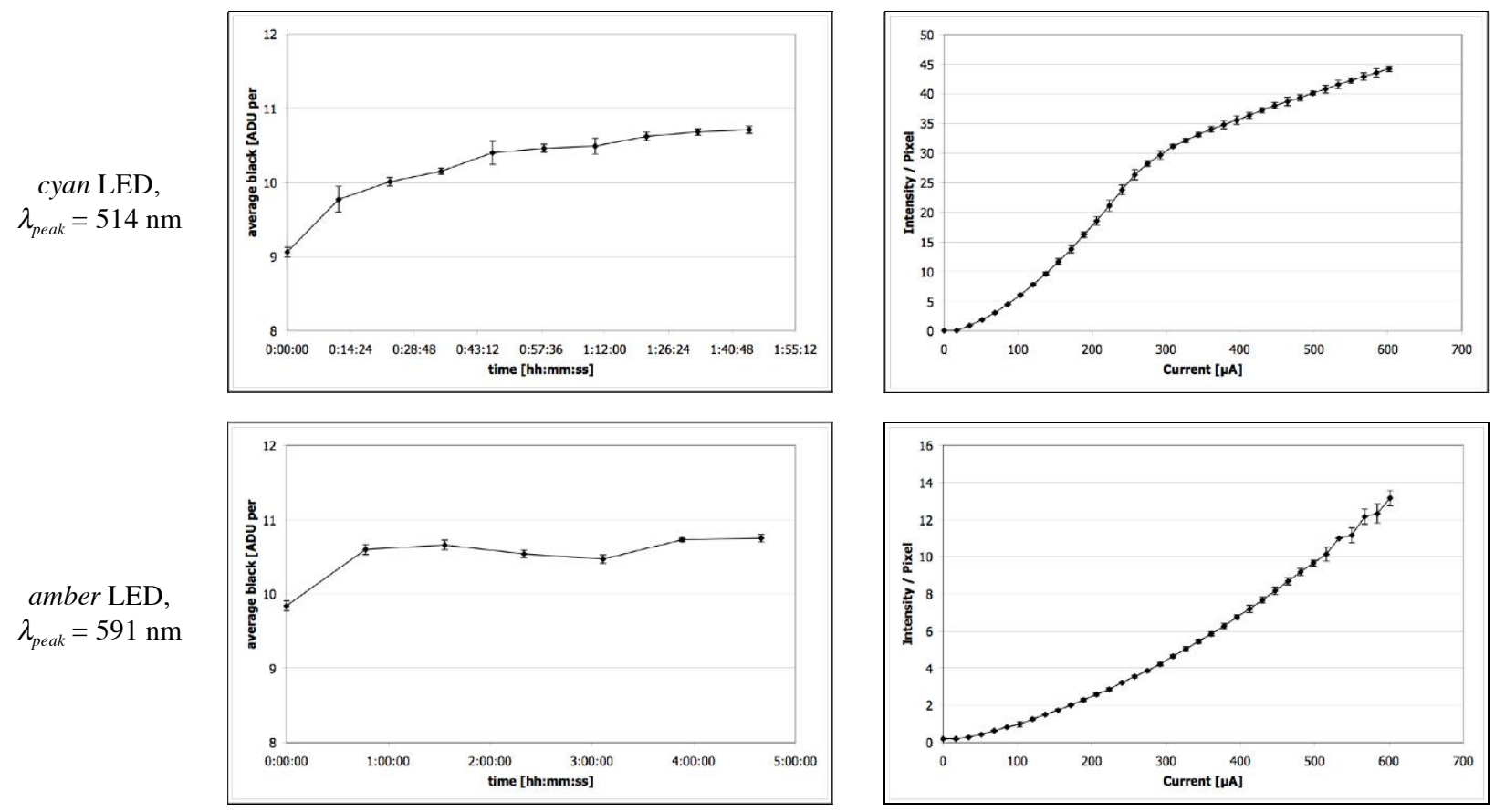

(a) Change in "black" background as a function of time. Each data point is the average value $\pm 3 \sigma$

(b) Range of calibration curves of repeated measurements associated with the various time intervals. Each data point is the average value $\pm \sigma$

Figure 6: Stability of the calibration curves over two-hour (cyan LED) and five-hour (amber LED) time intervals.

While there is a slight drift of the "black" image background over the indicated time interval, this is less than one grey level which-given the 8-bit analog-to-digital converter (ADC) in the frame grabber-represents an uncertainty of about $0.4 \%$. This is negligible compared to the variations associated with biochemical (i.e. staining) procedures.

The procedure for calibration based upon electrons, i.e. current, is straightforward. Consider two systems where we wish to make a comparison of the intensities measured in one versus the intensities measured in the other. The difference between the two systems might be:

- $\quad$ measurements on the same exact system at two different times;

- measurements made where one or more components of the system have been changed, e.g. objective lens, camera integration time, camera binning, optical filters, digital camera

- $\quad$ measurements made on two different microscope systems in one laboratory

- measurements made at two different laboratories

For each system and preceding each measurement, a calibration curve such as those shown in Figures 5 is made using the LED that is matched to the fluorophore. (See Table 2 above.) The calibration curves are then presented and used as shown in Figure 7. The axes in Figure 7 have been swapped with respect to the axes in Figures 5 and $6 \mathrm{~b}$. For a given measured intensity in a specific pixel, the curve indicates the equivalent electrical current. As current can be accurately and precisely measured, this means that we can now compare the currents between the two systems.

If, for example, the pixel intensity is grey value 61, then for System \#1 this corresponds to a current of $480 \mu \mathrm{A}$ and for System \#2 only $96 \mu \mathrm{A}$. System \#1, therefore, is configured in such a way that it requires $5 \mathrm{x}$ as much current to produce an equivalent grey value intensity. This might correspond to an integration time that is $5 \mathrm{x}$ shorter, $200 \mathrm{~ms}$ as compared to $1000 \mathrm{~ms}$. Once the scale factor between the two systems is known for each intensity/current level, the actual microscope sample can be measured and translated to units of current. 


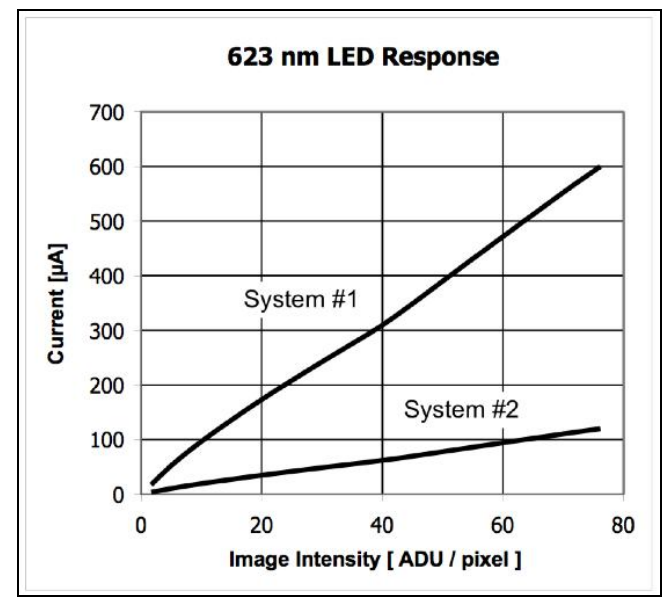

Figure 7: Calibration curves for two "independent" systems. The red LED is considered as the appropriate color to mimic a cell that has been stained with a "red" fluorophore as listed in Table 2. The horizontal axis represents a system-specific relative measure and the vertical axis represents an absolute measure.

The fluorescence excitation source can also be calibrated by this procedure. Using an appropriate configuration (e.g. filters, lenses, electro-optics, etc.), a "clear-field" (background) image can be recorded to determine how much excitation light is reaching the specimen plane and then the image acquisition system. This intensity value can then be translated into current and monitored for changes due to aging or system configuration.

\section{CALIBRATION WITH PHOTONS}

While the calibration procedure described above may be correct, it may not be as intuitively appealing as describing intensity measures of light with units of light. To accomplish this we need to be able to translate the electrons into photons. To accomplish this we need an additional measuring device, a radiometer, that is capable of measuring the absolute power of the light that falls on the face of the camera that is used in the microscope system. In our work we have used an Ophir radiometer (Ophir Optronics, <http://www.ophiropt.com>) and a Newport radiometer model 480 (Newport Corp. <http://www.newport.com>). The results reported below were acquired with the Ophir system.

The theory is as follows. The total energy $E_{o}$ from an LED can be written as:

$$
E_{o}=\int_{0}^{\infty} E(\lambda) d \lambda=P_{o} \Delta T
$$

where $E(\lambda)$ is the spectral distribution of the LED, $P_{o}$ is the optical power produced by the LED as measured by the radiometer at the camera face, and $\Delta T$ is the measurement (integration) time. But this energy is caused by $N$ photons that hit the face of the camera with $n(\lambda)$ photons per wavelength. These can be related by:

$$
E(\lambda)=\frac{h c}{\lambda} n(\lambda) \quad \Rightarrow \quad n(\lambda)=\frac{\lambda}{h c} E(\lambda)
$$

where $h=$ Planck's constant and $c$ is the speed of light. It follows that:

$$
N=\int_{0}^{\infty} n(\lambda) d \lambda=\int_{0}^{\infty} \frac{\lambda}{h c} E(\lambda) d \lambda=\frac{1}{h c} \int_{0}^{\infty} \lambda E(\lambda) d \lambda
$$

The average wavelength $\langle\lambda\rangle$, whose measured value we have given in Table 1 , is defined as:

$$
<\lambda>=\frac{\int_{0}^{\infty} \lambda E(\lambda) d \lambda}{\int_{0}^{\infty} E(\lambda) d \lambda} \Rightarrow \int_{0}^{\infty} \lambda E(\lambda) d \lambda=<\lambda>\int_{0}^{\infty} E(\lambda) d \lambda=<\lambda>P_{o} \Delta T
$$


The conclusion is that the number of photons that strike the face of the camera is:

$$
N=\frac{P_{o}<\lambda>\Delta T}{h c}
$$

As we shall see, this power $P_{o}$ (per pixel) is linearly related to the recorded intensity (per pixel) as $P_{o}=\alpha \cdot I$. where $\alpha$ is a to-be-calculated scale factor. We now measure the power delivered to the face of the camera versus the recorded intensity to produce the graph shown in Figure 8.

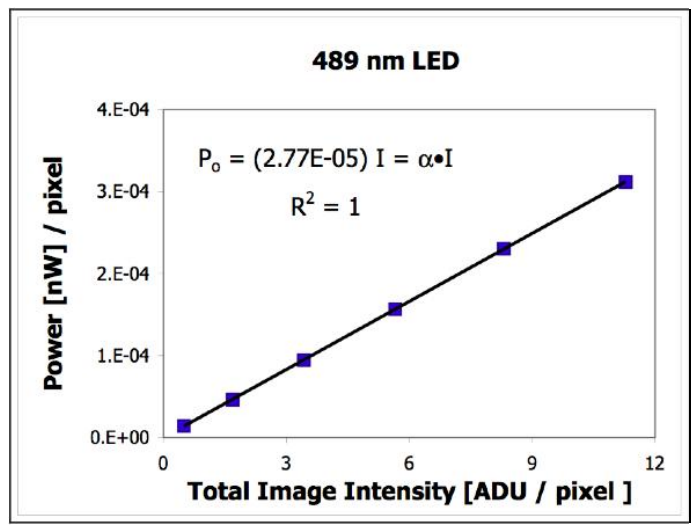

Figure 8: Conversion of image intensity to LED power in $[\mathrm{nW}]$, at the face of the camera. The data for the blue LED, $\lambda_{\text {peak }}=$ $477 \mathrm{~nm}$ are shown.

It is clear that the LED spectral power and the intensity are linearly related. The slope for the curve shown in Figure 8 is $\alpha=2.77 \times 10^{-5}$ [nW/ADU]. This value varies from one LED to another but the functional relationship is always linear. For the blue LED with $\langle\lambda\rangle=489 \mathrm{~nm}$, the camera with an integration time of $40 \mathrm{~ms}$, and the $\alpha$ determined above, we have:

$$
N=\frac{P_{o}<\lambda>\Delta T}{h c}=\left(\frac{\alpha<\lambda>\Delta T}{h c}\right) I=2729 \cdot I\left[\frac{\text { photons }}{\text { ADU }}\right]
$$

Thus a change from, for example, grey value 203 to 204 represents an additional 2729 photons from the blue LED. With the use of a radiometer to measure the LED spectral power at the face of the camera, the calibration paradigm can now move from electrons per pixel to photons per pixel as depicted in Figure 9.
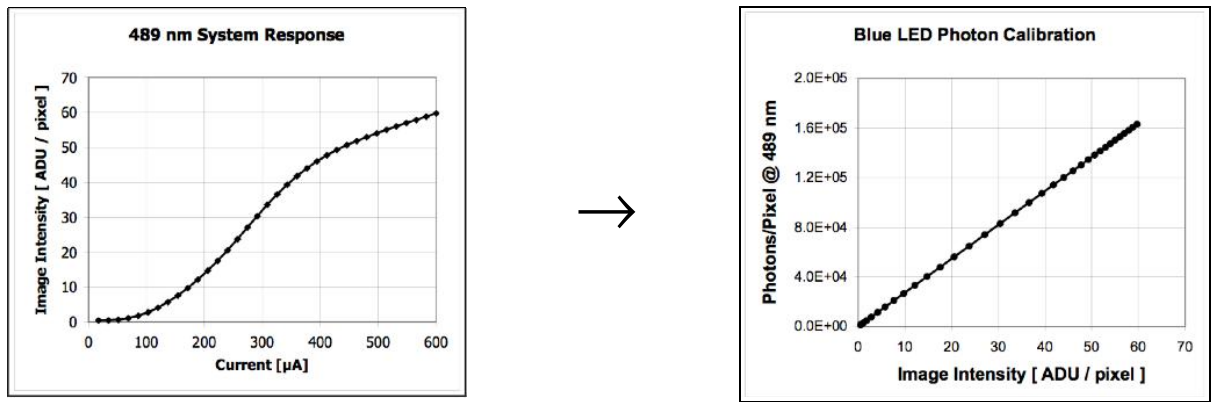

Figure 9: The intensity value at a given pixel can be described as the number of photons produced by a color-equivalent LED.

\section{SUMMARY AND CONCLUSIONS}

We have shown that it is feasible to produce absolute fluorescence calibration in electrons using an LED Microscope Slide that we have designed, built and tested. Further, the calibration can also be made in photons if one has access to a standard laser radiometer. The calibration can take into account the effect of many parameters that vary from one quantitative microscope to another. These parameters can include: magnification, numerical aperture, wavelength, glass 
transmission, excitation light source variation, quantum efficiency, pixel size, fill factor, binning, integration time, and ADC gain and offset ${ }^{4}$.

Our approach is based upon LEDs mounted upon a printed-circuit board that is about the size of a microscope slide. Each LED "simulates" a fluorescing cell with the color of the LED chosen to mimic the color of the desired fluorophore. Computer control of the current to the LED corresponds to changing the amount of light-the "fluorescence" - of the simulated cell. The measure of the light acquired in a given integration period is then the electrons that have been used to produce that light in that time interval. The radiometer provides the additional information required to transform the calibration units from electrons (per pixel) to photons (per pixel).

We are not the first to consider mechanisms for calibrating fluorescence microscopy nor are we the first to use LEDs to do so. Beach and Duling ${ }^{5}$ and Beach $^{6}$ have long been advocates of this approach. Model and Burkhardt ${ }^{7}$ have considered using standardized amounts of fluorescent dye in solution. Others have used the QuantiBRITE beads mentioned earlier.

Our contribution is a complete, compact, portable, inexpensive system that is durable and easy to use. In future work we will be comparing our results to the QuantiBRITE beads and collecting results over a variety of quantitative microscope systems.

\section{ACKNOWLEDGMENTS}

This work was partially supported by the Bsik Research Program Cyttron, the research program Nanophoresis of the Netherlands Foundation for Fundamental Research in Matter (FOM) and the Delft Research Center Life Science and Technology.

\section{REFERENCES}

${ }^{1}$ K. Lennartz, M. Lu, M. Flasshove et al., "Improving the biosafety of cell sorting by adaptation of a cell sorting system to a biosafety cabinet," Cytometry A, 66:2, pp. 119-27, 2005.

${ }^{2}$ C. Vogt, A. Losche, S. Kleinsteuber et al., "Population profiles of a stable, commensalistic bacterial culture grown with toluene under sulphate-reducing conditions," Cytometry A, 66:2, pp. 91-102, 2005.

${ }^{3}$ N. Watanabe, Y. H. Wang, H. K. Lee et al., "Hassall's corpuscles instruct dendritic cells to induce CD4+CD25+ regulatory T cells in human thymus," Nature, 436:7054, pp. 1181-5, 2005.

${ }^{4}$ I.T. Young, J.J. Gerbrands, and L.J. van Vliet, "Image Processing Fundamentals," in The Digital Signal Processing Handbook, V.K. Madisetti and D.B. Williams ed., pp. 51.1 - 51.81, CRC Press in cooperation with IEEE Press, Boca Raton, Florida, 1998.

${ }^{5}$ J. M. Beach and B. R. Duling, “A light-emitting diode light standard for photo- and videomicroscopy,” J Microsc, 172 (Pt 1), pp. 41-8, 1993.

${ }^{6}$ J.M. Beach, “A LED light calibration source for dual-wavelength microscopy,” Cell Calcium, 21:1, pp. 63-68, 1997.

${ }^{7}$ M.A. Model and J.K. Burkhardt, "A Standard for Calibration and Shading Correction of a Fluorescence Microscope," Cytometry, 44:4, pp. 309-316, 2001. 